\title{
Mechanical and Electrical Models for Piezoelectric Dispersions in Oriented Polymers
}

\author{
Eiichi Fukada and Munehiro Date \\ The Institute of Physical and Chemical Research \\ Yamato, Saitama, Japan.
}

(Received March 30, 1970)

\begin{abstract}
A complex piezoelectric modulus $d^{*}=d^{\prime}-j d^{\prime \prime}$ is defined as a ratio of electrical polarization to mechanical stress. In a number of oriented polymers such as polyglutamates and in polarized piezoelectric ceramic powders molded in resins, characteristic temperature variations of $d^{\prime}$ and $d^{\prime \prime}$ have been observed. $d^{\prime}$ takes a maximum and $d^{\prime \prime}$ changes from positive to negative at a temperature where the thermal relaxations in mechanical and dielectric properties take place.

The externally applied stress produces a mechanical strain of crystallites, which is accompanied by piezoelectric polarization. The strain of crystallites is influenced by mechanical relaxation in amorphous regions surrounding the crystallites. The polarization in crystallites induces polarization observed on the surfaces of the specimen. The induced polarization is also affected by the dielectric relaxation in amorphous regions surrounding the crystallites. Therefore the phase difference between the applied stress and the resulting polarization is the sum of viscoelastic and dielectric phase differences.

A mechanical model consisting of a spring for crystallites and a spring and two dashpots for amorphous regions is proposed. An electric circuit consisting of a capacitance for crystallites and a capacitance and two resistances for amorphous regions is also proposed. The combination of the two models gives a qualitative representation of the piezoelectric dispersion curves.
\end{abstract}

KEY WORDS Complex Piezoelectric Modulus/Piezoelectric Dispersion / Viscoelastic / Dielectric / Mechanical Model / Thermal Relaxation / Oriented Polymers /

It has been established that electrical polarization is produced by applying shear stress in a number of oriented films of crystalline polymers such as cellulose and poly $(\gamma$-methyl-L-glutamate). ${ }^{1,2}$ A complex piezoelectric modulus $d^{*}=d^{\prime}-j d^{\prime \prime}$ has been defined as a ratio of the alternating electrical polarization to the alternating shear stress. ${ }^{3}$ An apparatus for measuring $d^{\prime}$ and $d^{\prime \prime}$ in a wide range of temperatures has also been developed. ${ }^{4}$ An interesting variation of piezoelectric moduli with temperatures has been reported in different kinds of polymers. In order to explain such piezoelectric temperature dispersion, a mechanical model and an electrical circuit will be proposed in this paper.

\section{PIEZOELECTRIC TEMPERATURE DISPERSION}

Temperature variation of piezoelectric moduli in oriented $\operatorname{poly}(\boldsymbol{\gamma}$-methyl-L-glutamate $)$ is illustrated in Figure $1 .^{5}$ The measurement was carried out at $20 \mathrm{~Hz}$. The molecular conformation of the polymer is $\alpha$-helical. The piezoelectric modulus $d_{25}{ }^{*}$ represents electrical polarization on the film surface produced by a unit magnitude of shear stress in the film plane. The modulus $d_{25}$ ' shows a component of polarization which is in phase with the applied stress. The latter modulus is related to the difference of phase angle between electrical polarization and mechanical stress. The phase angle $\delta$ is represented by $\tan \delta=d_{25}{ }^{\prime \prime} / d_{25}{ }^{\prime}$. The expression is similar to the complex elastic compliance, where the imaginary component relates to the difference of phase angle between mechanical strain and mechanical stress.

The modulus $d_{25}{ }^{\prime}$ increases up to about $0^{\circ} \mathrm{C}$ and then decreases with temperature. The modulus $d_{25}{ }^{\prime \prime}$ alters its sign from positive to negative around temperatures at which $d_{25}{ }^{\prime}$ 

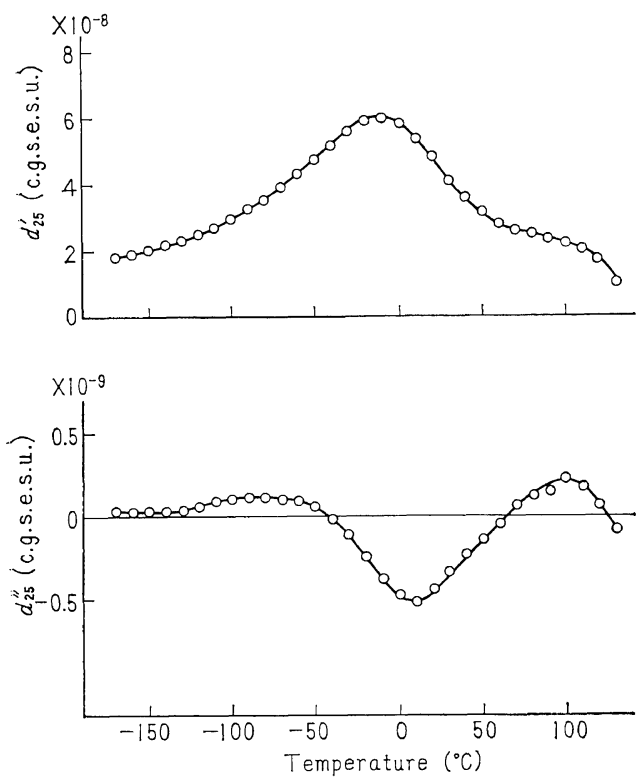

Figure 1. Temperature variation of real piezoelectric modulus $d_{25}{ }^{\prime}$ and imaginary piezoelectric modulus $d_{25}{ }^{\prime \prime}$ for a film of poly( $\gamma$-methyl-L-glutamate) elongated to twice the original length.

becomes maximum. The sign of $d_{25}{ }^{\prime \prime}$ indicates that the polarization lags behind the stress at low temperatures and leads beyond the stress at high temperatures. It has been known that the thermal motion of side chains takes place near $00^{\circ} \mathrm{C}$ in poly $(\gamma$-methyl-L-glutamate $){ }^{6}$ The variation of $d_{25}{ }^{\prime}$ and $d_{25}{ }^{\prime \prime}$ is also seen around $100^{\circ} \mathrm{C}$, where the thermal motion of disordered main chains takes place. ${ }^{7}$

Piezolectric polymers are usually well crystallized and uniaxially oriented. Oriented crystallites are embedded in amorphous regions of the polymer. If the crystallites are strained under external stress, electrical polarization will be produced in them. It is therefore necessary to be concerned with a mixture of piezoelectric crystallites and a non-piezoelectric medium.

In order to make a model experiment, mixtures of piezoelectric crystals and amorphous polymer were prepared. Fine powders of piezoelectric certamics (PZT) were uniformly mixed into equivolume polyester resin with hardener and the mixture was left at room temperature for a day to complete curing. A

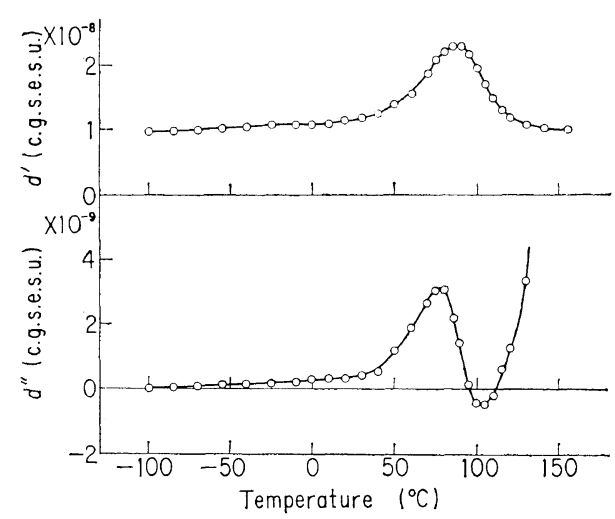

Figure 2. Temperature variation of real piezoelectric modulus $d^{\prime}$ and imaginary piezoelectric modulus $d^{\prime \prime}$ for a mixture of PZT ceramic powders and polyester resin, which has been polarized under a direct electctric field.

molded film thus produced with a thickness of about $0.1 \mathrm{~mm}$ was subjected to a static high voltage of about $1000 \mathrm{~V}$ for a few hours at room temperature. After such polarization process, the film acquired piezoelectric properties. Figure 2 illustrates the temperature dispersion curve of piezoelectric moduli for an equivolume mixture of ceramic and polyster. The tensile stress in the plane of the mixture film produced an electrical polarization normal to the film surface. The modulus $d^{\prime}$ shows a maximum at about $80^{\circ} \mathrm{C}$ and $d^{\prime \prime}$ varies with temperature through a maximum and a minimum in the neighbourhood of $80^{\circ} \mathrm{C}$. The Curie point of PZT ceramics is about $300^{\circ} \mathrm{C}$, while the glass transition of this polyester takes place at about $80^{\circ} \mathrm{C}$, where the distinct dispersion is observed as in Figure 2. The behaviour of piezoelectric dispersion for the mixture of piezoelectric ceramic powders and non-piezoelectric polymer is very similar to that observed in oriented semicrystalline polymers as illustrated in Figure 1.

The results described above support our view that electrical polarization is principally caused in piezoelectric crystallites, that thermal relaxation in amorphous regions surrounding the crystallites results in changes of effective mechanical stress acting on the crystallites, and that thus the strain or polarization of crystallites changes with the variation of temperature. 


\section{E. Fukada and M. Date}

\section{A MECHANICAL MODEL}

A simple four-elements model will be proposed in order to explain piezoelectric dispersion. The model is shown in Figure 3. Piezoelectric crystallites are represented by a spring, whose elastic stiffness is $G_{c}$. Non-piezoelectric amorphous regions surrounding the crystallites are represented by two dashpots and a spring. A dashpot with viscosity coefficient $\eta_{p}$ is connected in parallel with the spring $G_{\mathrm{c}}$. The other dashpot with viscosity coefficient $\eta_{s}$ is connected in series with the spring $G_{\mathrm{c}}$. A spring with stiffness $G_{\mathrm{a}}$ represents the elasticity in amorphous regions.

We assume that piezoelectric polarization is caused by the strain of the spring $G_{c}$. Let us consider first the variation of the strain $\gamma_{\mathrm{c}}$ of the spring $G_{\mathrm{c}}$ with a rise of temperature when the externally applied stress $\sigma$ is kept constant. If the dashpot $\eta_{p}$ moves, the stress in $G_{\mathrm{c}}$ increases and the strain $\gamma_{c}$ of the piezoelectric spring increases. The phase of $\gamma_{c}$, then, lags behind that of the applied stress $\sigma$. Therefore piezoelectric polarization increases with temperature and its phase lags behind the stress. If the dashpot $\eta_{s}$ moves, the stress in $G_{\mathrm{a}}$ increases and the stress in $G_{\mathrm{c}}$ decreases, and accordingly the strain $\gamma_{c}$ decreases from the initial value. The phase of $\gamma_{c}$ now leads beyond that of the applied stress $\sigma$, if the alternating stress and strain are concerned.

The piezoelectric modulus $d_{E}{ }^{*}=K_{\gamma_{\mathrm{c}}} / \sigma$ is easily calculated as a function of angular frequency $\omega$ on the basis of the model in Figure 3, where

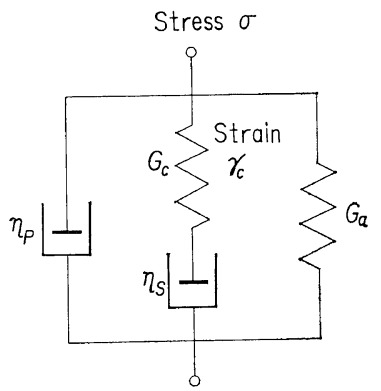

Figure 3. A mechanical model for showing the influence of viscoelastic relaxation on temperature and frequency dispersions of piezoelectric moduli in polymers.
$K$ is a coefficient between the piezoelectric modulus and the strain of crystallites, in other words, the piezoelectric polarization per unit stress in crystallites. The result is

$$
d_{E}{ }^{*}=d_{E}{ }^{\prime}-j d_{E}{ }^{\prime \prime}
$$

$$
\begin{aligned}
d_{E}{ }^{\prime} & =\frac{K}{G_{\mathrm{a}}} \\
& \times \frac{1+\left(G_{\mathrm{c}} / G_{\mathrm{a}}\right)+\left(\tau_{p} / \tau_{s}\right)}{\left[1+\left(G_{\mathrm{c}} / G_{\mathrm{a}}\right)+\left(\tau_{p} / \tau_{s}\right)\right]^{2}+\left[\omega \tau_{p}-\left(1 / \omega \tau_{s}\right)\right]^{2}}
\end{aligned}
$$

$$
\begin{aligned}
d_{E}{ }^{\prime \prime} & =\frac{K}{G_{\mathrm{a}}} \\
& \times \frac{\omega \tau_{p}-\left(1 / \omega \tau_{s}\right)}{\left[1+\left(G_{\mathrm{c}} / G_{\mathrm{a}}\right)+\left(\tau_{p} / \tau_{s}\right)\right]^{2}+\left[\omega \tau_{p}-\left(1 / \omega \tau_{s}\right)\right]^{2}} \\
& \tan \delta_{E}=\frac{d_{E}^{\prime \prime}}{d_{E}^{\prime}}=\frac{\omega \tau_{p}-\left(1 / \omega \tau_{s}\right)}{1+\left(G_{\mathrm{c}} / G_{\mathrm{a}}\right)+\left(\tau_{p} / \tau_{s}\right)}
\end{aligned}
$$

where the following two relaxation times are introduced

$$
\begin{aligned}
\tau_{p} & =\eta_{p} / G_{\mathrm{a}} \\
\tau_{s} & =\eta_{s} / G_{\mathrm{c}}
\end{aligned}
$$

We further define the following parameters

$$
\begin{gathered}
\alpha=G_{\mathrm{c}} / G_{\mathrm{a}}=v_{\mathrm{c}} \boldsymbol{G}_{\mathrm{c} 0} / v_{\mathrm{a}} G_{\mathrm{a} 0} \\
\beta=\eta_{p} / \eta_{s}=v_{\mathrm{a} p} \eta_{\mathrm{a} 0} / v_{\mathrm{a} s} \eta_{\mathrm{a} 0} \\
\alpha \beta=\tau_{p} / \tau_{\mathrm{s}}
\end{gathered}
$$

where, $G_{\mathrm{c} 0}$ and $G_{\mathrm{a} 0}$ are stiffness per unit volume for crystalline and non-crystalline regions. $\alpha$ is proportional to the ratio of the volume fraction of crystallites $v_{\mathrm{c}}$ to the volume fraction of amorphous regions $v_{\mathrm{a}}$. $\eta_{\mathrm{a} 0}$ is the viscosity coefficient per unit volume of amorphous regions. $v_{\mathrm{a} p}$ and $v_{\mathrm{as}}$ are volume fractions of amorphous regions which are located in parallel and in series with respect to crystallites respectively. $\beta$ is larger if the fraction of amorphous regions connected in parallel with crystallites is larger.

Employing these parameters, eq 2, 3, and 4 are simplified as follows

$$
\begin{gathered}
d_{E}{ }^{\prime} / d_{E m}^{\prime}=1 /\left(1+x^{2}\right) \\
d_{E}{ }^{\prime \prime} / d_{E m}^{\prime}=x /\left(1+x^{2}\right) \\
\tan \delta_{E}=x
\end{gathered}
$$

where, 


$$
\begin{gathered}
x=\frac{\omega \tau_{p}-\left(\alpha \beta / \omega \tau_{p}\right)}{1+\alpha+\alpha \beta} \\
d_{m}{ }^{\prime}=\frac{K / G_{\mathrm{a}}}{1+\alpha+\alpha \beta}
\end{gathered}
$$

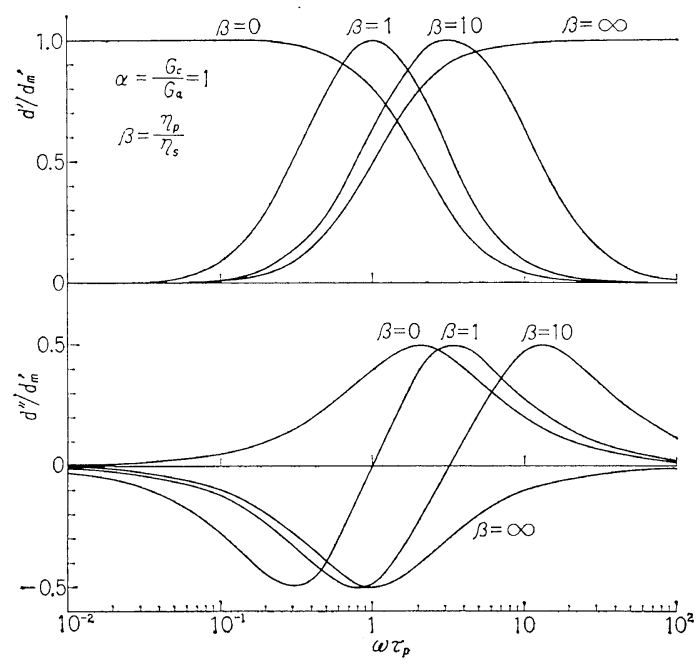

Figure 4. The variation of piezoelectric moduli $d^{\prime}$ and $d^{\prime \prime}$ with angular frequency $\omega$ and temperature $T$, calculated by means of the mechanical model in Figure 3.

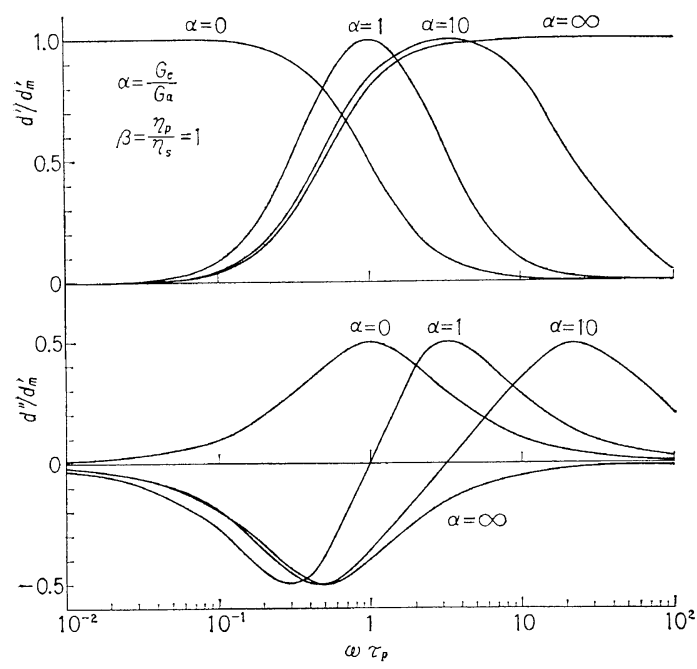

Figure 5. The variation of piezoelectric moduli $d^{\prime}$ and $d^{\prime \prime}$ with angular frequency $\omega$ and temperature $T$, calculated by means of the mechanical model in Figure 3.
The curves of $d_{E}{ }^{\prime} / d_{E m}^{\prime}$ and $d_{E}{ }^{\prime \prime} / d_{E m}^{\prime}$ as functions of $x$ or $\omega \tau_{p}$ are thus easily calculated numerically and are illustrated in Figures 4 and 5. The abscissa in figures is the logarithm of $\omega \tau_{p}$. The viscosity coefficient in amorphous regions is usually expressed by an equation as $\eta_{\mathrm{a} 0}=\overline{\eta_{\mathrm{a} 0}}$ $\exp (\Delta E / R T)$, where $\overline{\eta_{\mathrm{a} 0}}$ is a constant and $\Delta E$ the activation energy for viscosity and $R$ the gas constant and $T$ the temperature. Therefore, the relaxation time $\tau_{p}$ can also be expressed by $\tau_{p}=\tau_{p 0} \exp (\Delta E / R T)$, where $\tau_{p 0}$ is a constant including $G_{\mathrm{a}}$ and $\overline{\eta_{\mathrm{a} 0}}$. The abscissa in Figures 4 and 5 is thus $A+\ln \omega+\Delta E / R T$, where $A$ is a constant. Therefore, the curves in Figures 4 and 5 represent the variations of $d_{E}{ }^{\prime}$ and $d_{E}{ }^{\prime \prime}$ with either $\ln \omega$ or $1 / T$. The shape of the curve depends upon the values of parameters $\alpha$ and $\beta$.

It is assumed in Figure 4 that $\alpha=G_{\mathrm{c}} / G_{\mathrm{a}}=1$, that is, the apparent elastic stiffnesses in crystalline and amorphous regions are about the same. If the fraction of amorphous regions in parallel with crystallites predominates over that in series, so that $\eta_{p} \gg \eta_{s}$ and $\beta \approx \infty, d_{E}^{\prime}$ increases with $\omega$, and decreases with $T$, and $d_{E}{ }^{\prime \prime}$ is in the leading phase. If the reverse holds, that is, $\eta_{p} \ll \eta_{s}$ and $\beta \approx 0, d_{E}{ }^{\prime}$ decreases with $\omega$ and increases with $T$, and $d_{E}{ }^{\prime \prime}$ is in the lagging phase. In between the foregoing two extremes, $\eta_{p} \approx \eta_{s}$ and $\beta \approx 1$, $d_{E}{ }^{\prime}$ shows a maximum against $\omega$ or $T$ and $d_{E}{ }^{\prime \prime}$ shows a lagging maximum at a lower temperature range and a leading minimum at a higher temperature range.

In Figure 5 the assumption is made that $\beta=\eta_{p} / \eta_{s}=1$, that is, the apparent viscosity coefficients or the volume fractions of amorphous regions in parallel and in series with the crystallites are almost the same. If the degree of crystallinity is large, so that $G_{\mathrm{c}} \gg G_{\mathrm{a}}$ and $\alpha \approx \infty, d_{E}{ }^{\prime}$ increases with $\omega$ and decreases with $T$ and ${d_{E}}^{\prime \prime}$ has a minimum with leading phase. If the degree of crystallinity is small, so that $G_{\mathrm{c}} \ll G_{\mathrm{a}}$ and $\alpha \approx 0, d_{E}{ }^{\prime}$ decreases with $\omega$ and increases with $T$ and $d_{E}{ }^{\prime \prime}$ has a maximum with lagging phase. In the intermediate condition, $G_{\mathrm{c}}=G_{\mathrm{a}}$ and $\alpha=1$, the curve is the same as that in Figure 4 with $\alpha=\beta=1$.

It is concluded that the shape of curves markedly depends on the distribution of 


\section{E. Fukada and M. Date}

amorphous regions between parallel and series connections with crystallites and also on the degree of crystallinity. When $G_{\mathrm{c}}>G_{\mathrm{a}}$ and $\eta_{p}>\eta_{s}, d_{E}{ }^{\prime}$ gradually decreases with temperature and $d_{E}$ " takes only a leading phase. Observations corresponding to this case have been reported in oriented films of cellulose triacetate ${ }^{8}$ and optically active poly(propylene oxide). ${ }^{9}$ On the other hand, when $G_{\mathrm{c}}<G_{\mathrm{a}}$ and $\eta_{p}<\eta_{s}, d_{E}{ }^{\prime}$ gradually increases with temperature and $d_{\mathrm{E}}{ }^{\prime \prime}$ takes only a lagging phase. Observations corresponding to this case have also been reported in dried plates of wood $^{4}$ and oriented films of cellulose diacetate. ${ }^{8}$ The conditions $G_{\mathrm{c}} \approx G_{\mathrm{a}}$ and $\eta_{p} \approx \eta_{s}$ seem to be most common in many oriented piezoelectric polymers. The $d_{E}{ }^{\prime}$ takes a maximum and $d_{E}{ }^{\prime \prime}$ takes a maximum with a lagging phase followed by a minimum with a leading phase with a rise of temperature. The examples are shown in Figure 1 for poly( $\gamma$-methyl-L-glutamate) and in Figure 2 for the mixture of ceramic powders and polyester resin.

\section{AN ELECTRICAL MODEL}

Under the externally applied stress, the crystallites are strained and piezoelectric polarization takes place on the surfaces of crystallites. This polarization induces the electrical charge on the outer surfaces of the specimen where the electrodes are joined. Since crystallites are embedded in the amorphous regions, the dielectric properties in the latter regions must influence the polarization induced on the surfaces of the specimen. In order to take this situation into account, a simple electric circuit is proposed in addition to the mechanical model explained above.

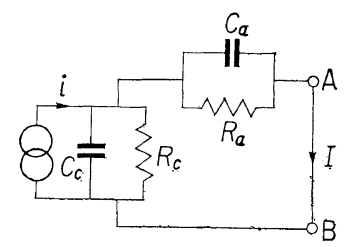

Figure 6. An electrical circuit for showing the influence of dielectric relaxations on the temperature and frequency dispersions of piezoelectric moduli in polymers.
Figure 6 shows an equivalent circuit representing the combined structure of crystallites. and surrounding amorphous regions. The electric capacitors with capacitance $C_{\mathrm{c}}$ and $C_{\mathrm{a}}$ represent the crystallites and amorphous regions. respectively. The electric resistances $R_{\mathrm{c}}$ and $R_{\mathrm{a}}$ indicate the electric leakages connected in parallel with crystallites and with amorphous regions respectively. Points $\mathrm{A}$ and $\mathrm{B}$ represent the surfaces of the specimen, the electric impedance between which is assumed to consist essentially of a series combination of $C_{\mathrm{c}}$ and $C_{\mathrm{a}}$. Under an applied stress $\sigma$, the capacitor $C_{\mathrm{c}}$ is polarized by $p$. This implies connecting an electric source with a constant current $i$ to the capacitor $C_{\mathrm{c}}$. The electric current $I$ thereby induced between points $\mathrm{A}$ and $\mathrm{B}$ is proportional to the polarization $P$ on the surfaces of the specimen.

The influence of the dielectric absorption in amorphous regions will be estimated by calculating the ratio $P / p$ by means of an electrical circuit in Figure 6 . We define quantities $d_{\varepsilon}{ }^{\prime}$ and $d_{\varepsilon}^{\prime \prime}$ as

$$
P / p=I / i=d_{\varepsilon}{ }^{\prime}-j d_{\varepsilon}{ }^{\prime \prime},
$$

where suffix $\varepsilon$ is added to show the contribution of dielectric absorption in amorphous regions. on the piezoelectric moduli.

A simple calculation gives the following equations.

$$
\begin{gathered}
d_{\varepsilon}{ }^{\prime}=\frac{1+R_{\mathrm{a}} / R_{\mathrm{c}}+\omega^{2}\left(R_{\mathrm{a}} C_{\mathrm{a}}\right)^{2}\left(1+C_{\mathrm{c}} / C_{\mathrm{a}}\right)}{\left(1+R_{\mathrm{a}} / R_{\mathrm{c}}\right)^{2}+\omega^{2}\left(R_{\mathrm{a}} C_{\mathrm{a}}\right)^{2}\left(1+C_{\mathrm{c}} / C_{\mathrm{a}}\right)^{2}} \\
d_{\varepsilon}{ }^{\prime \prime}=\frac{\omega\left(R_{\mathrm{c}} C_{\mathrm{c}}-R_{\mathrm{a}} C_{\mathrm{a}}\right) R_{\mathrm{a}} / R_{\mathrm{c}}}{\left(1+R_{\mathrm{a}} / R_{\mathrm{c}}\right)^{2}+\omega^{2}\left(R_{\mathrm{a}} C_{\mathrm{a}}\right)^{2}\left(1+C_{\mathrm{c}} / C_{\mathrm{a}}\right)^{2}}
\end{gathered}
$$

$$
\begin{aligned}
& \tan \delta_{\varepsilon}=d_{\varepsilon}{ }^{\prime \prime} / d_{\varepsilon}{ }^{\prime} \\
& =\frac{\omega\left(R_{\mathrm{c}} C_{\mathrm{c}}-R_{\mathrm{a}} C_{\mathrm{a}}\right) R_{\mathrm{a}} / R_{\mathrm{c}}}{1+\left(R_{\mathrm{a}} / R_{\mathrm{c}}\right)+\omega^{2}\left(R_{\mathrm{a}} C_{\mathrm{a}}\right)^{2}\left(1+C_{\mathrm{c}} / C_{\mathrm{a}}\right)}
\end{aligned}
$$

Let us define a relaxation time $\tau_{\varepsilon}$ as follows

$$
\tau_{\varepsilon}=\frac{C_{\mathrm{c}}+C_{\mathrm{a}}}{\left(1 / R_{\mathrm{c}}\right)+\left(1 / R_{\mathrm{a}}\right)}
$$

Eq. 15 and 16 are then simplified as follows

$$
d_{\varepsilon}^{\prime}=d_{\varepsilon 0}^{\prime}+\left(d_{\varepsilon \infty}^{\prime}-d_{\varepsilon 0}^{\prime}\right) \frac{\omega^{2} \tau_{\varepsilon}{ }^{2}}{1+\omega^{2} \tau_{\varepsilon}^{2}}
$$


Models for Piezoelectric Dispersions in Polymers

$$
d_{\varepsilon}^{\prime \prime}=-\left(d_{\varepsilon \infty}^{\prime}-d_{\varepsilon 0}^{\prime}\right) \frac{\omega \tau_{\varepsilon}}{1+\omega^{2} \tau_{\varepsilon}^{2}}
$$

where

$$
d_{\varepsilon 0}^{\prime}=R_{\mathrm{c}} /\left(R_{\mathrm{c}}+R_{\mathrm{a}}\right)
$$

and

$$
d_{\varepsilon \infty}^{\prime}=C_{\mathrm{a}} /\left(C_{\mathrm{c}}+C_{\mathrm{a}}\right)
$$

The following relations also hold.

$$
d_{\varepsilon \infty}^{\prime}-d_{\varepsilon 0}^{\prime}=\frac{R_{\mathrm{a}} C_{\mathrm{a}}-R_{\mathrm{c}} C_{\mathrm{c}}}{\left(C_{\mathrm{c}}+C_{\mathrm{a}}\right)\left(R_{\mathrm{c}}+R_{\mathrm{a}}\right)}=2 d_{\varepsilon \max }^{\prime \prime}
$$

If the leakage resistance surrounding or inside the crystallites is very large, $R_{\mathrm{c}} C_{\mathrm{c}}>R_{\mathrm{a}} C_{\mathrm{a}}$, and hence according to eq $23, d_{\varepsilon \infty}^{\prime}<d_{\varepsilon 0}^{\prime}$. The sign of $d_{\varepsilon}^{\prime \prime}$ in eq 20 is positive, that is, the phase of the observed piezoelectric polarization lags behind that of the polarization on the crystallites. On the other hand, if the leakage resistance surrounding or inside the crystallites is very small, $R_{\mathrm{c}} C_{\mathrm{c}}<R_{\mathrm{a}} C_{\mathrm{a}}$ and hence $d_{\varepsilon \infty}^{\prime}>d_{\varepsilon 0}^{\prime}$. The sign of $d_{\varepsilon}{ }^{\prime \prime}$ is now negative and the phase of the piezoelectric polarization observed leads beyond that of the polarization on the crystallites.

If we introduce parameters $a=C_{\mathrm{c}} / C_{\mathrm{a}}$ and $b=R_{\mathrm{a}} / R_{\mathrm{c}}$, eq 21 and 22 become

$$
d_{\varepsilon 0}^{\prime}=1 /(1+b)
$$

$$
d_{\varepsilon \infty}^{\prime}=1 /(1+a)
$$

where $a$ is related to the volume ratio of crystalline regions to amorphous regions. The values of $d_{\varepsilon}{ }^{\prime}$ and $d_{\varepsilon}{ }^{\prime \prime}$ have been calculated as a function of $\omega \tau_{\varepsilon}$ and are shown in Figure 7 and Figure 8. The abscissa indicates $\log \omega+\Delta E^{\prime} / R T$ $+A^{\prime}$, where $A^{\prime}$ is a constant and $\Delta E^{\prime}$ is an activation energy for dielectric relaxation.

In Figure $7, a=C_{\mathrm{c}} / C_{\mathrm{a}}=1$ is assumed, so that the volume fractions of the crystalline and the amorphous regions are about the same. If $b=R_{\mathrm{a}} / R_{\mathrm{c}} \ll 1, d_{\varepsilon}^{\prime}$ increases with $T$ and $d_{\varepsilon}{ }^{\prime \prime}$ takes a lagging phase. If $b=1, d_{\varepsilon}{ }^{\prime}$ is a constant of $1 / 2$ and $d_{\varepsilon}{ }^{\prime \prime}=0$. If $b \gg 1, d_{s}{ }^{\prime}$ decreases with $T$ and $d_{\varepsilon}^{\prime \prime}$ takes a leading phase.

In Figure $8, b=R_{\mathrm{a}} / R_{\mathrm{c}}=1$ is assumed, so that the leakage resistances in both the amorphous regions and in the crystalline regions are the same. If the degree of crystallinity is small and $a=C_{\mathrm{c}} / C_{\mathrm{a}} \ll 1, d_{\varepsilon}{ }^{\prime}$ decreases with $T$ and $d_{\varepsilon}{ }^{\prime \prime}<0$, if $a=1, d_{\varepsilon}{ }^{\prime}=1 / 2$ and $d_{\varepsilon}{ }^{\prime \prime}=0$. If the degree of crystallinity is large and $a \gg 1, d_{\varepsilon}{ }^{\prime}$ increases with $T$ and $d_{\varepsilon}^{\prime \prime}>0$.

In most dry specimens the situation $R_{\mathrm{c}} C_{\mathrm{c}}>$ $R_{\mathrm{a}} C_{\mathrm{a}}$ and hence $a / b>1$ holds. Therefore, $d_{\varepsilon}^{\prime}$ decreases with $\omega$. and increases with $T$, and $d_{\varepsilon}^{\prime \prime}$ takes a lagging phase. However, in the case when the surface leakage of crystallites is large, for instance, when the specimen contains large

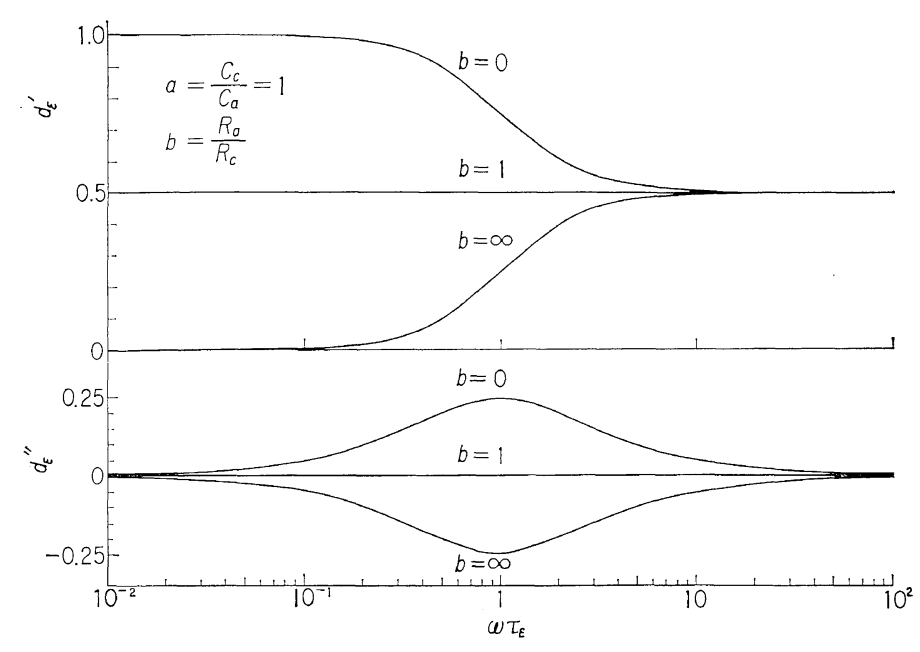

Figure 7. The variation of $d_{\varepsilon}{ }^{\prime}$ and $d_{\varepsilon}{ }^{\prime \prime}$, the ratio of observable polarization to the polarization on crystallites, with angular frequency $\omega$ and temperature $T$, calculated by means of the electrical circuit in Figure 6. 


\section{E. FuKadA and M. DATE}

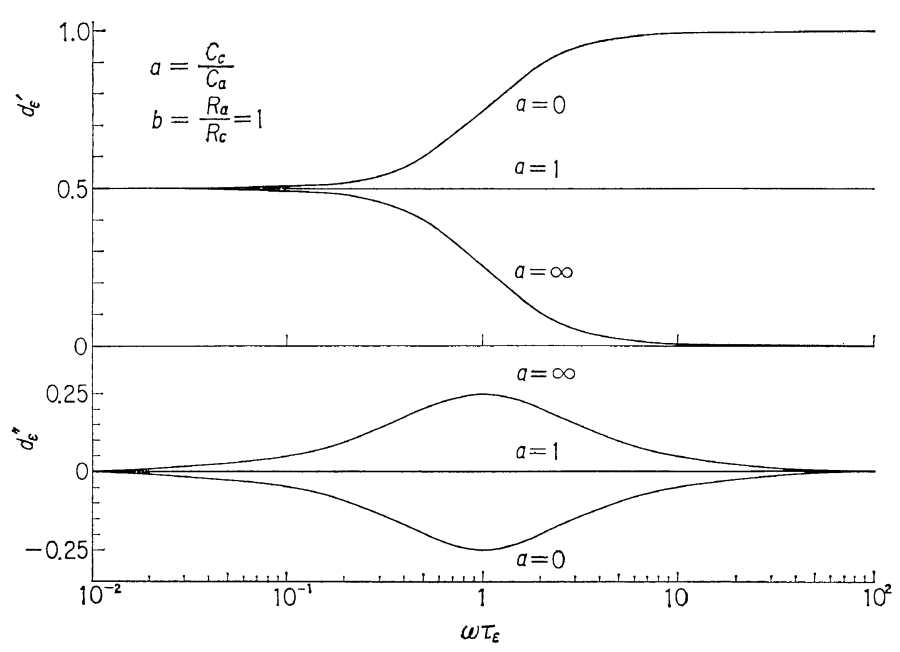

Figure 8. The variation of $d_{\varepsilon}^{\prime}$ and $d_{\varepsilon}{ }^{\prime \prime}$ with angular frequency $\omega$ and temperature $T$, calculated by means of the electrical circuit in Figure 6.

amounts of moisture, the condition $R_{\mathrm{c}} C_{\mathrm{c}}<R_{\mathrm{a}} C_{\mathrm{a}}$ and hence $a / b<1$ holds. Then $d_{\varepsilon}{ }^{\prime}$ increases with $\omega$ and decreases with $T$, and $d_{\varepsilon}^{\prime \prime}$ takes a leading phase.

\section{DISCUSSION}

Our basic idea for explaining piezoelectric dispersion is that an externally applied stress produces a mechanical strain in piezoelectric crystallites inside the specimen, which is accompanied by an electric polarization of the crystallites, and that the polarization in the crystallites induces electrical polarization in the specimen, which is experimentally observed. Because most polymers are viscoelastic, there are always time lags between the applied mechanical stress and the resulting strain and also between the applied electric field and the resulting polarization. Piezoelectricity is a combination of mechanical and electrical properties and therefore, the time difference between the applied stress and the resulting polarization should be caused by both mechanical and dielectric origins. In the case of inverse piezoelectricity, there is also a time difference between the applied electric field and the resulting mechanical strain.

In the preceding section, the electrical polarization produced by unit external stress was given by

$$
p / \sigma=K_{\curlyvee \mathrm{c}} / \sigma=d_{E}{ }^{*}=d_{E}{ }^{\prime}-j d_{E}{ }^{\prime \prime}
$$

The observed polarization $\boldsymbol{P}$ produced by polarization $p$ in the crystallites was given by

$$
P / p=d_{\varepsilon}{ }^{*}=d_{\varepsilon}{ }^{\prime}-j d_{\varepsilon}{ }^{\prime \prime}
$$

The overall piezoelectric modulus, that is, the ratio of the polarization externally observed to the applied stress is given by

$$
\begin{aligned}
P / \sigma & =d^{*}=d^{\prime}-j d^{\prime \prime} \\
& =\left(d_{E}{ }^{\prime}-j d_{E}{ }^{\prime \prime}\right)\left(d_{\varepsilon}{ }^{\prime}-j d_{\varepsilon}{ }^{\prime}\right) \\
& =\left(d_{E}{ }^{\prime} d_{\varepsilon}{ }^{\prime}-d_{E}{ }^{\prime \prime} d_{\varepsilon}{ }^{\prime}\right)-j\left(d_{E}{ }^{\prime}{ }^{\prime} d_{\varepsilon}{ }^{\prime}+{d_{E}}{ }^{\prime} d_{\varepsilon}{ }^{\prime \prime}\right)
\end{aligned}
$$

Further rearrangements give

$$
\begin{gathered}
d^{\prime}=d_{E}{ }^{\prime} d_{\varepsilon}{ }^{\prime}\left(1-\tan \delta_{E} \tan \delta_{\varepsilon}\right) \\
d^{\prime \prime}=d_{E}{ }^{\prime \prime} d_{\varepsilon}{ }^{\prime}\left[1+\left(\tan \delta_{\varepsilon} / \tan \delta_{E}\right)\right] \\
\tan \delta=\left(\tan \delta_{E}+\tan \delta_{\varepsilon}\right) /\left(1-\tan \delta_{E} \tan \delta_{\varepsilon}\right)
\end{gathered}
$$

Generally the value of $\tan \delta_{E}$ is in the order of magnitude $10^{-1}$ and the value of $\tan \delta_{\varepsilon}$ is usually one order of magnitude less than that of $\tan \delta_{E}$. Therefore $\tan \delta_{E} \tan \delta_{\varepsilon}$ can be ignored against unity. Eq 27 and 29 are simplified as

$$
\begin{gathered}
d^{\prime}=d_{E}{ }^{\prime} d_{\varepsilon}{ }^{\prime} \\
\tan \delta=\tan \delta_{E}+\tan \delta_{\varepsilon}
\end{gathered}
$$


It is of interest to note that the phase factor $\tan \delta$ for piezoelectric dispersion is the sum of those for viscoelastic dispersion and for dielectric dispersion.

The theoretical calculation for piezoelectric dispersion should be undertaken by making a product of $d_{E}{ }^{*}$ and $d_{\varepsilon}{ }^{*}$ as a function of angular frequency $\omega$ and temperature $T$. In many real polymers, however, the dielectric loss is usually smaller than the mechanical loss. The contribution of elastric loss predominates over that of dielectric loss.

The present models were proposed only for predicting the qualitative variation of piezoelectric moduli with temperature and frequency. If the models are completed the viscoelastic and dielectric dispersion curves must also be derived from the models. The mechanical model in Figure 3, however, does not yield the viscoelastic dispersion in amorphous regions owing to the limited numbers of spring and dashpot. The electrical circuit in Figure 6 will give a dielectric dispersion for interfacial polarization at the boundaries between the crystallites and the amorphous regions, but it does not show the dielectric dispersion only in amorphous regions. If increased numbers of elements are introduced in Figures 3 and 6 , it would be possible to derive the viscoelastic and dielectric dispersion curves as well as the piezoelectric dispersion curve. The calculation would then be much more complex and the use of a computer would be preferable.

The present models do not take into consideration the distribution of relaxation times in mechanical and dielectric properties either. The aim of the present paper is the presentation of a basic idea, without going into a complicated mathematical calculation, which enables us to explain the piezoelectric dispersion on the basis of viscoelastic and dielectric relaxations in amorphous regions surrounding the crystallites.

Acknowledgment. The authors thank Mr. M. Oshiki for assistance in experiments.

\section{REFERENCES}

1. E. Fukada, Biorheology, 5, 199, (1968).

2. E. Fukada, Ultrasonics, 6, 229, (1968).

3. R. Holland, IEEE Trans. Sonics. Ultrasonics $S U-14,1,18,(1967)$.

4. E. Fukada, M. Date, and K. Hara, Japan. J. Appl. Phys., 8, 151, (1969).

5. M. Date, S. Takashita, and E. Fukada, J. Polym. Sci., Part A-2, 8, 61, (1970).

6. S. Sugai, K. Kamashima, S. Makino, and J. Noguchi, J. Polym. Sci., Part A-2, 4, 183, (1966).

7. Y. Hashino, M. Yoshino, and K. Nagamatsu, Rep. Prog. Polym. Phys. Japan., 9, 297, (1966).

8. E. Fukada, M. Date, and T. Emura, J. Soc. Mat. Sci. Japan., 17, 335, (1968).

9. T. Furukawa and E. Fukada, Nature, 221, 1235, (1969). 